\title{
Structure design and analysis of Magnetic Levitation
}

\section{Motor}

\author{
Zhida Zhu ${ }^{\mathrm{a}, *}$, Li Zeng ${ }^{\mathrm{a}}$, Fan Zhang ${ }^{\mathrm{a}}$, Jin Sun ${ }^{\mathrm{a}}$, Lifeng Zhang ${ }^{\mathrm{b}}$ \\ ${ }^{\mathrm{a} C}$ College of Mechanical Engineering, Yangzhou University, Yangzhou 225127,China \\ ${ }^{\mathrm{b}}$ Department of Electrical Engineering and Electronics, Kyushu Institute of Technology, Fukuoka, Japan
}

\begin{abstract}
This paper introduced the working principle of magnetic levitation motor, and the design of the overall structure of a magnetic levitation motor. Then, according to relevant literature, the paper determined the parameters of the various parts of magnetic bearing by calculation and ANSYS simulation. After analyzing the distribution of magnetic lines, the magnetic flux density, and air-gap magnetic field situation, it optimized the design of magnetic levitation motor.
\end{abstract}

Keywords: magnetic levitation, permanent magnet motor, finite element, structure design.

\section{Introduction}

With the development of industrial modernization, people constantly improve to the requirement of motor, not only require motor can support huge torque but also ask it can get high speed running, even super speed. When motor is in high speed moving, however, the friction resistance and heat problem produced by mechanical bearing friction seriously hindered the development of high-speed motor ${ }^{(1)}$, the presence of the magnetic suspension bearing solved this problem. Using electromagnetic force, magnetic suspension bearing can make motor rotor suspending in the air with no contact which not only realized motor high speed moving but also radically solved mechanical friction problem. This paper designed a magnetic levitation bearing for permanent magnet motor, not only calculated the structural parameters of magnetic bearings, and using the ANSYS software to electromagnetic analysis and structure optimization.

\section{The overall structure of the magnetic suspension permanent magnet motor}

The structure of magnetic suspension permanent magnet motor see figure 1.magnetic levitation bearing of permanent magnet motor mainly consist of radial permanent magnet bearings, axial electromagnetic bearing and permanent magnet motor. Radial permanent magnet bearings 1 and 2, on both sides of motor with symmetrical distribution, and axial electromagnetic bearing work together make motor rotor suspended running. In the ends of the motor is equipped with two mechanical bearings 5 and 6. There is clearance in the radial and axial of mechanical bearing and motor rotor, radial air gap value and axial air gap value is respectively less than the axial magnetic bearing and radial magnetic bearing air gap value which to protective motor and magnetic bearing when the load is too large .Motor parts use permanent magnet synchronous motor, mainly to the external output torque.

\section{Structure calculation and analysis of radial permanent magnet bearings}

\subsection{Structure calculation of radial permanent magnet bearings}

Radial permanent magnet bearings can be roughly divided into suction type and repulsion type. In the structure of the suction type of magnetic bearing, magnetic field itself in magnetic ring reduces at the adjacent side of both static magnetic ring and active magnetic ring. In the structure of repulsive type of magnetic bearing, the position of magnetic ring is different from magnetization direction. The permanent magnetic field of the magnet increases the strength of its adjacent demagnetization magnetic field ${ }^{(2)}$, which is the reason of using repulsive type of permanent magnet bearing here. The material of permanent magnet ring use M42M. Besides, while assembling inside and outside magnetic ring of permanent magnetic bearing left and right sides are not aligned and there exists a small offset, which is in order to make two permanent magnet bearings in axial as stable as possible. 


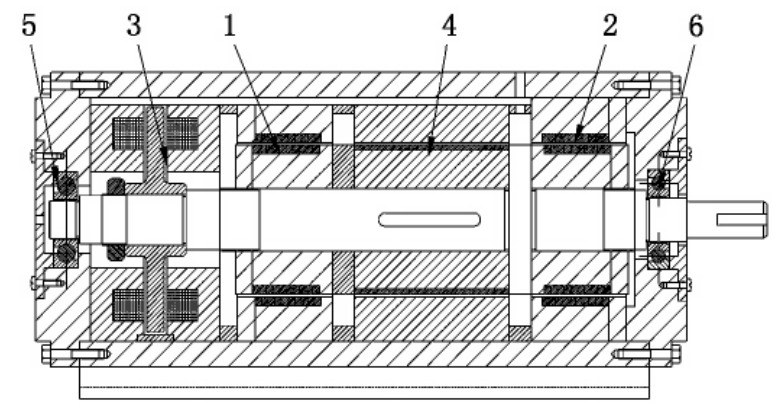

1,2- Radial permanent magnet bearings. 3- Axial permanent magnetic bearing. 4- Permanent magnetic motor. 5,6- Mechanical bearings.

Figure.1 Structure of magnetic suspension permanent magnet motor

Radial magnetic bearing is composed of multiple superimposed magnetic rings. According to the different magnetization directions, the calculation of magnetic ring size can be divided into two cases of radial or axial magnetization. This paper uses the type of axial magnetization. Here is to calculate the size of the single pair of magnetic rings. Set the magnetic induction intensity for magnetic circuit $B_{\mathrm{m}}$, magnetic field intensity $H_{m}$, length of a magnetic path $L_{m}$, cross-sectional area $S_{m}$, magnetic field intensity of working air gap $B_{g}$, magnetic field intensity of working air gap $H_{g}$, length of a magnetic path of working air gap $L_{g}$, cross-sectional area of working air gap $S_{g}$, thickness of support $\omega_{0}$.

According to Ampere circuital theorem ${ }^{(3)}$, in the outside air of magnetic ring and rotor magnetic circuit, there is:

$$
H_{m} L_{m}=H_{\text {rotor }} L_{\text {rotor }}+H_{\text {air }} L_{\text {air }}
$$

In magnetic circle of the working air gap between the magnetic rings, there is:

$$
H_{m} L_{m}=H_{a i r}^{\prime} L_{a i r}+H_{g} L_{g}
$$

Among which: $H_{\text {rotor }}$ is the magnetic field intensity of rotor magnetic ring, $H_{\text {air }}$ is the magnetic field intensity of air outside the magnetic rings, $H_{\text {air }}^{\prime}$ is the magnetic field intensity of working air gap between magnetic rings. $L_{\text {rotor }}$ is the length of the circular magnetic circuit of rotor,
$L_{\text {air }}$ is the length of the magnetic circuit in the air of magnetic rings.

The magnetic field strength in the air and the rotor is equivalent to magnetic field in the air gap. According to the distribution of the magnetic field of magnetic circuit, it is found that they roughly meet such a relationship:

$$
\begin{gathered}
H_{\text {air }}=\frac{1}{4} H_{g}, H_{\text {rotor }}=\frac{1}{3} H_{g}, H_{\text {air }}^{\prime}=\frac{1}{5} H_{g}, L_{g}=\frac{1}{3} L_{m}, \\
B_{m} S_{m}=\sigma \mu_{0} H_{g} S_{g}, L_{\text {air }}=2 \omega_{0}, L_{\text {rotor }}=L_{m}+2 \omega_{0} . \text { Add }
\end{gathered}
$$

the above conditions to eqn. 1 and eqn.2, there is:

$$
2 H_{m} L_{m}=\frac{47}{30} \frac{B_{m} S_{m} \omega_{0}}{\sigma \mu_{0} S_{g}}+\frac{2}{3} \frac{B_{m} S_{m} L_{m}}{\sigma \mu_{0} S_{g}}
$$

Among which: $\sigma$ is field leakage coefficient.

$\sigma=4.5 \cdot \mu_{0}$ is permeability of vacuum.

$\mu_{0}=4 \pi \times 10^{-7} \mathrm{H} / \mathrm{m}$.

Assuming the static working point of permanent magnet is at the best working point. The parameters of N24M are known. $B_{m}=0.661 T, L_{g}=0.5 \mathrm{~mm}$,

$H_{m}=497 \mathrm{kA} / \mathrm{m}, \omega_{0}=3 \mathrm{~mm}$. It is required that the air-gap field $B_{g}$ should reach 1T-1.4T. Then get the physical dimension of permanent rings: $L_{m}=6.44 \mathrm{~mm}$, use $L_{m}=6 \mathrm{~mm}$.

\subsection{Electromagnetic analysis of radial permanent magnet bearings}

The magnetic ring stacking number and when inside and outside magnetic rings have no axial and radial offsets, use the $2 \mathrm{~d}$ finite element model to carry out analysis.

Distribution of magnetic field lines of multi-layer magnetic rings axial magnetization is shown in figure 2 . When multi-layer magnetic rings axial magnetization, the lines of magnetic force are well distributed, and mainly distributed in the up and low ends of magnetic rings. In addition, there is also a phenomenon the magnetic leakage is larger at the end of a rotating shaft. Figure 3 is the radial magnetic bearing magnetic clouds. Figure 3 shows that when the superimposed magnetic ring begins axial 
magnetization, magnetic induction intensity in the permanent magnet is between $0.55 \mathrm{~T}-0.75 \mathrm{~T}$, more close to the best working point of permanent magnet, magnetic induction intensity between work air gaps of the magnetic rings is between $1.0 \mathrm{~T}-1.3 \mathrm{~T}$, within the scope of the designed value, and distributed symmetrically and uniformly.

Mainly, the magnetic induction intensity of air gap is distributed on the outer surface of the outer and inner magnetic rings, basically distributed is symmetry. The magnetic induction intensity of air gap is shown in figure 4 .

Besides, the air-gap magnetic field $B_{g}$ shown in figure 4 is a smaller value, just because it shows the air gap magnetic field value on the edge of bearing end. The actual value at the air gap can be achieved through the magnetic figure in magnetic clouds figure.

Based on the above analysis, finally determine the permanent magnet ring $L_{m}=6 \mathrm{~mm}$, height of magnetic ring $h=7 \mathrm{~mm}$. The electromagnetic performance analysis of permanent magnet ring shows it has nothing to do with its radius. So here select the outer diameter within the magnet ring $d_{1}=22 \mathrm{~cm}$, inner diameter $d_{2}=20.7 \mathrm{~cm}$, inner diameter of outer magnet ring $d_{3}=23 \mathrm{~cm}$, outer diameter $d_{4}=24.40 \mathrm{~cm}$.The capacity of permanent magnetic radial bearing $F_{1}$ can be calculated by equation (4):

$$
F_{1}=\frac{B_{g}^{2} A_{0}}{\mu_{0}}
$$

Among which: $A_{0}$ is section area of air gap, $A_{0} \approx 0.00138 m^{2}$. Set $B_{g}=1.3 T$. Add related parameters to equation (4) and calculate to $F_{1}=1891.72 \mathrm{~N}$.

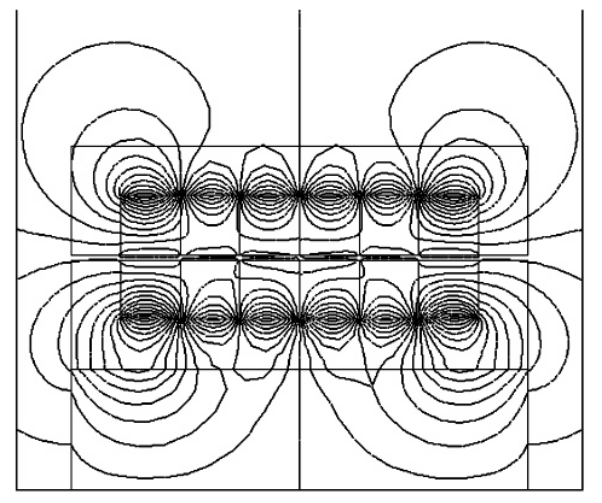

Fig.2 Distribution lines of magnetic force when multilayer magnetic rings' axial magnetization.

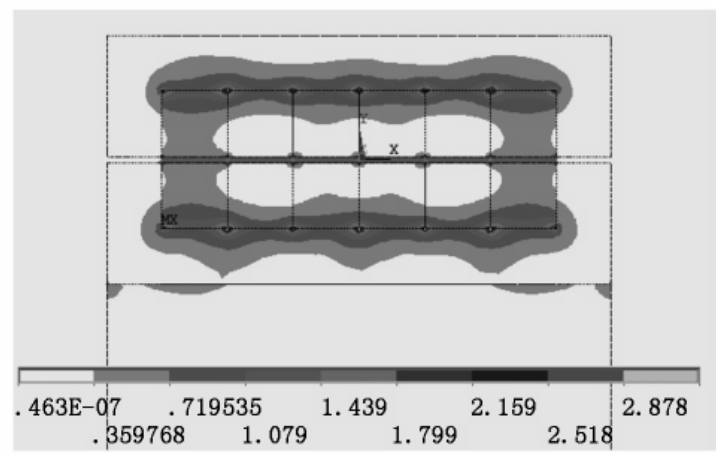

Fig.3 Flux density cloud figure the radial magnetic bearing

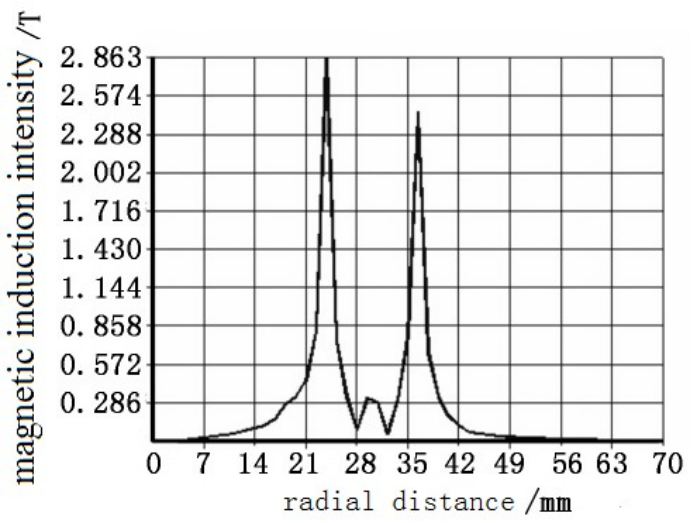

Fig.4 The flux density curve of the superimposed magnet ring of air gap

\section{The structure calculation and analysis of the axial electromagnetic bearing}

\subsection{The structure calculation of the axial electromagnetic bearing}

The axial magnetic bearing structure as shown in figure 5 , the rotor disk is made of whole material electrical pure iron. In order to make full use of material, adopting the principle of same magnetic resistance, that is mean every 
magnetic poles area is equal. In figure $5, D$ is the axial magnetic bearing stator outer diameter, $D_{1}$ is axial magnetic bearing stator inner diameter, $L$ is the width of the stator, $\mathrm{x}$ is the air gap between the stator and rotor, $A_{1}$,

$A_{2}$ is the area of the two magnetic circuit respectively,

their area all is $A$. The theory calculation formulas of structure design of axial magnetic bearing are as follows:

$$
\left\{\begin{array}{l}
F_{2}=\frac{\lambda^{2} J^{2} \mu_{0}}{4 \chi^{2}} A A_{c u}^{2} \\
A_{c u} \leq \frac{2 B_{s} \chi}{\lambda J \mu_{0}} \\
A=\pi a(D-a) \\
A_{c u}=\frac{\left(\pi L \sqrt{D_{1}^{2}+4 A / \pi}-2 A\right)\left(\sqrt{D^{2}+4 A / \pi}-\sqrt{D_{1}^{2}+4 A / \pi}\right)}{2 \pi \sqrt{D_{1}^{2}+4 A / \pi}}
\end{array}\right.
$$

The parameters and dimensions of the axial magnetic bearings are as follows: Space factor $\lambda=0.7$,current density $J=3 \mathrm{~A} / \mathrm{mm}^{2}$, The axial magnetic bearing magnetic pole area $A=10990 \mathrm{~mm}^{2}, x=0.6 \mathrm{~mm}$, $D_{1}=190 \mathrm{~mm}, D=290 \mathrm{~mm}$, the thickness of the disk of the stator $a=35 \mathrm{~mm}, L=75 \mathrm{~mm}$,electrician pure ferromagnetic saturation induction intensity $B_{s}=1.50 T$, coil cavity area $A_{c u}=682.43 \mathrm{~mm}^{2}$. Bringing above parameters into formula (5),calculating the bearing capacity of the axial magnetic bearings is $F_{2}=5.201 \times 10^{3} N$

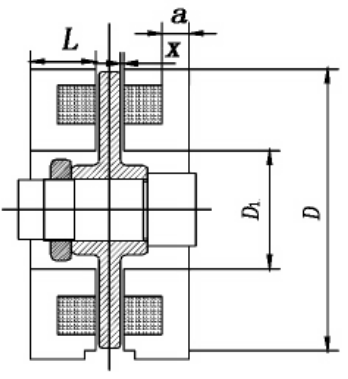

(a) The eletromagnetic bearing structure

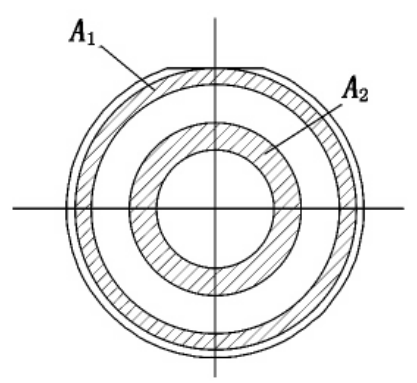

(b) Section of bearing stat
Fig.5 The structure of axial magnetic bearing

\subsection{The electromagnetic analysis of axial magnetic}

\section{bearing structure}

Under the finite element software ANSYS analysis the electromagnetic field of axial magnetic bearing stator and rotor and got distribution diagram of main parameters of electromagnetic characteristics: the lines of magnetic force, magnetic induction intensity contours, magnetic field intensity vector. The entire field is made up of 4 parts: stator, rotor, shaft, and the air gap ${ }^{(4)}$.Due to axial magnetic bearings diagram is axial symmetric graphics, therefore, just need take a quarter section of cross section to analysis and bring related boundary conditions and structure parameters, respectively, into the finite element software ANSYS, calculating the electromagnetic properties of axial magnetic bearings. After calculating clouds of lines of magnetic force distribution and the magnetic induction intensity distribution are got, as figure 6 and figure 7 is shown. Figure 6 and figure 7 show clearly the magnetic distribution: (1) Axial magnetic bearings of magnetic induction intensity in the middle of the stator and rotor magnetic circuit and the air gap distribution more uniform, in the air gap edge and corner part of the field lines is sparse, magnetic induction intensity is weak.(2) Axial magnetic bearings have magnetic leakage phenomenon, mainly to the leak in the coil, in fact if there is no limit boundary condition, also should through the rotor disc to the spindle axis of leakage and leakage to the outer space. For convenient of calculating the magnetic field, in the practical application of ANSYS software analysis, assuming that the border without magnetic flux ${ }^{(5)}$, so there is no leakage occurring. (3)Despite the magnetic leakage is existence, but, no matter in which direction, the leak is not large, so in the structural design of magnetic bearings, assuming no magnetic leakage is feasible.

\section{Copyright}

Papers submitted to ICISIP must describe original and unpublished researches. They should not have been submitted to other conferences or journals. Any paper which is under review elsewhere will not be considered for publication. Once an eligible paper is accepted, it will be published under a Creative Commons Attribution 3.0

\section{Conclusions}

This article through the design of radial permanent magnet bearings and axial magnetic bearing to replace the 
mechanical bearing, so that to meet the needs of permanent magnet motor suspension high-speed operation. According to the related theory and parameters, the basic structure size of the magnetic bearing is computed , and magnetic field of magnetic bearing has been carried on the related electromagnetic analysis by use of ANSYS software which make structure was further optimized.
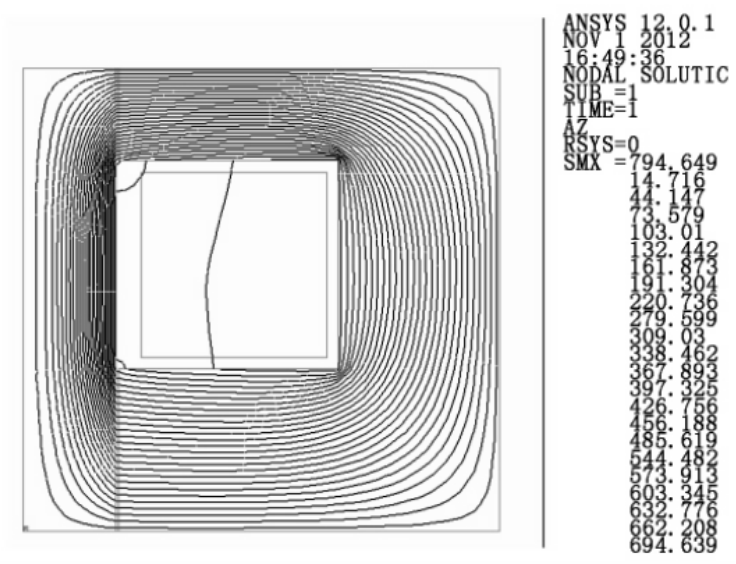

Fig.6 Distribution of the lines of magnetic force

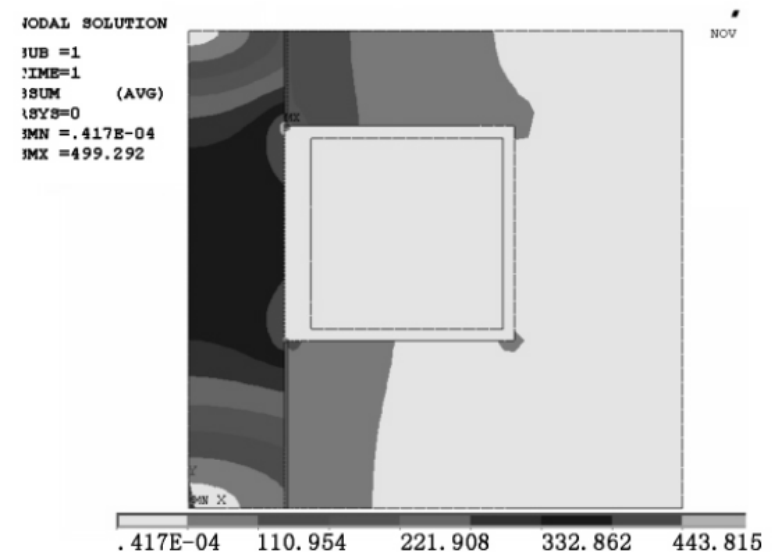

Fig.7 Induction intensity distribution of the radial magnetic bearing

\section{Acknowledgment}

This work was supported by China Natural Science Foundation for this project (Grant No. 51375427, 51475409), Jiangsu Province Basic Research Program Natural Science Foundation (Grant No.BY2015061-04, BK20141277), Yangzhou city - Yangzhou University of Science and Technology Cooperation Program Funds(No. SXT20140041, YZ2016244), 2016 Six talent peaks project in Jiangsu Province (No. JXQC-030).

\section{References}

(1) Deng Zhiquan and Yan Yangguang : "Basic theory and current study situation of no bearing ac motor", Transactions of China Electrotechnical Society, 2000,15(2):30-35.

(2) Xu Kaiguo : "Study and design of rotor of permanent magnetic levitation wind turbines”, Wuhan University of Technology Press, 2008:14-16.

(3) Tan Qingchang, Liu Mingjie and Meng Huiqin : "Calculation of carrying capacity and stiffness of permanent magnet centripetal bearing”, Journal of Tribology, 1994,14(4):337-344.

(4) Xie Longhan, Geng Yu and Qiu Wan : "ANSYS analysis of electromagnetic field”, Electronic Industry Press, 2012.

(5) $\mathrm{Hu}$ Yiefa, Zhou Zude, and Jiang Zhengfeng : "Basic theory and application of magnetic bearing”, China Machine Press, 2006. 